\title{
A NOTE TO THE REGULARITY OF SOLUTIONS FOR THE EVOLUTION P-LAPLACIAN EQUATIONS
}

JUNNING ZHAO*

In this note we consider the following Cauchy problem:

$$
\begin{cases}\frac{\partial u}{\partial t}=\operatorname{div}\left(|\nabla u|^{p-2} \nabla u\right) & (x, t) \in Q_{T}=R^{N} \times(0, T) \\ u(x, 0)=u_{0}(x) & x \in R^{N}\end{cases}
$$

where $p>2, u_{0} \in L_{\text {loc }}^{1}\left(R^{N}\right)$ satisfies that there exist constants $r>0$ and $\rho_{0}>0$ such that

$$
\left\|\left|u_{0} \|\right|_{r, \rho_{0}} \equiv \sup _{x_{0} \in R^{N}} \int_{B_{\rho_{0}}\left(x_{0}\right)}\left|u_{0}(x)\right| d x<\infty, \quad B_{\rho}\left(x_{0}\right)=\left\{\left|x-x_{0}\right|<\rho\right\} .\right.
$$

It is well known that there exists a solution $u \in C_{l o c}^{\alpha}\left(Q_{T}\right) \cap L_{l o c}^{\infty}\left(Q_{T}\right), \quad \nabla u \in$ $C_{l o c}^{\beta, \frac{\beta}{2}}\left(Q_{T}\right)$ to (1) (see [C],[DF],[DH]). The proofs of $\nabla u \in C_{l o c}^{\beta, \frac{\beta}{2}}\left(Q_{T}\right)$ are very complex and difficult. In this note we use another approach to prove the Hölder continuity of $\nabla u$. We prove $u_{t} \in L_{l o c}^{\infty}\left(Q_{T}\right), \quad \nabla u \in C_{l o c}^{\beta, \frac{\beta}{1+\beta}}\left(Q_{T}\right)$, where the Hölder index to t is great than $\frac{\beta}{2}$.

Definition. A function $u(x, t)$ defined in $Q_{T}$ is called a weak solution of $(1)$, if $u \in C_{l o c}^{\alpha}\left(Q_{T}\right) \cap L^{p}\left(0, T: W_{l o c}^{1, p}\left(R^{N}\right) \cap L^{\infty}\left(Q_{T}\right) \quad \alpha \in(0,1)\right.$ and for any $\phi(x, t) \in$ $C^{1}\left(\overline{Q_{T}}\right) \phi=0$ if $|x|$ large enough,

$$
\begin{aligned}
& \int_{R^{N}} u(x, t) \phi(x, t) d x+\int_{0}^{t} \int_{R^{N}}\left[-u \phi_{t}+|\nabla u|^{p-2} \nabla u \cdot \nabla \phi\right] d x d t \\
= & \int_{R^{N}} u_{0}(x) \phi(x, 0) d x .
\end{aligned}
$$

We obtain the following result.

Theorem 1. Let $u_{0} \geq 0, \|\left.\left|u_{0}(x)\right|\right|_{r, \rho_{0}}<\infty$ for some $\rho_{0}>0$. Then there exist constants $C>0$ and $\beta \in(0,1)$ such that the solution of (1) satisfies

$$
\sup _{R^{N}}\left|u_{t}(x, t)\right| \leq C t^{-\frac{N(p-1)+p}{\kappa}}\left\|\left|u_{0} \|\right|_{r, \rho}^{\frac{p}{\kappa}}, \quad \nabla u \in C^{\beta, \frac{\beta}{1+\beta}}\left(Q_{T}\right),\right.
$$

where $\kappa=N(p-2)+p$.

Proof. let $u$ be the solution of (1). Acoording to [WZYL], for $\forall \delta \in(0, T)$, $u(x, t+\delta)$ is the limit of the solutions of the following boundary value problems

$$
\begin{cases}\frac{\partial v}{\partial t}=\operatorname{div}\left(\left(|\nabla v|^{p-2}\right) \nabla v\right) & (x, t) \in B_{n} \times(0, T-\delta) \\ v(x, t)=u(x, \delta) & (x, t) \in \partial B_{n} \times(0, T-\delta) \\ v(x, 0)=u(x, \delta) & x \in B_{n}\end{cases}
$$


where $B_{n}=\{|x|<n\}$. Let $v_{n}$ be the solution of (5). Set

$$
w_{n}(x, t)=\lambda^{\gamma} v_{n}(x, \lambda t), \quad \lambda>1, \quad \gamma=\frac{1}{p-2} .
$$

Then $w_{n}$ satisfies

$$
\begin{cases}\frac{\partial w}{\partial t}=\operatorname{div}\left(|\nabla w|^{p-2} \nabla w\right) & (x, t) \in B_{n} \times\left(0, \frac{T-\delta}{\lambda}\right) \\ w(x, t)=\lambda^{\gamma} u(x, \delta) & (x, t) \in \partial B_{n} \times\left(0, \frac{T-\delta}{\lambda}\right) \\ w(x, 0)=\lambda^{\gamma} u(x, \delta) & x \in B_{n}\end{cases}
$$

Set $g_{n}=w_{n}-v_{n}$. By Comparison principle $g_{n} \geq 0$ and

$$
\begin{aligned}
& \int_{B_{n}} g_{n}(x, t) \phi(x, t) d x-\int_{0}^{t} \int_{B_{n}} g_{n} \phi_{t} d x d \tau+\int_{0}^{t} \int_{B_{n}}\left(\left|\nabla w_{n}\right|^{p-2} \nabla w_{n}-\right. \\
& \left.-\left|\nabla v_{n}\right|^{p-2} \nabla v_{n}\right) \cdot \nabla \phi d x d \tau=\int_{B_{n}}\left(\lambda^{\gamma}-1\right) u(x, \delta) \phi(x, 0) d x
\end{aligned}
$$

where $\phi \in C^{1}\left(\overline{B_{n} \times(0, T)}\right) \phi=0$ near $\partial B_{n}$. Notice that (see [DH])

$$
\|u(x, \delta)\|_{L^{\infty}\left(R^{N}\right)} \leq C \delta^{-\frac{N}{\kappa}}\left\|\mid u_{0}\right\|_{r, \rho_{0}}^{\frac{p}{\kappa}}
$$

In (7), we take

$$
\phi=\left(g_{n}-k\right)_{+} \quad k=\left(\lambda^{\gamma}-1\right)\|u(x, \delta)\|_{L^{\infty}\left(R^{N}\right)} .
$$

Using Steklov averaging process, we get

$$
\begin{aligned}
& \int_{B_{n}}\left(g_{n}-k\right)_{+}^{2} d x \\
& +2 \int_{0}^{t} \int_{B_{n} \cap\{w>k\}}\left(\left|\nabla w_{n}\right|^{p-2} \nabla w_{n}-\left|\nabla v_{n}\right|^{p-2} \nabla v_{n}\right)\left(\nabla w_{n}-\nabla v_{n}\right) d x d \tau=0 .
\end{aligned}
$$

This implies $g_{n} \leq k$ a.e. on $B_{n} \times\left(0, \frac{T-\delta}{\lambda}\right)$. Thus

$$
0 \leq \lambda^{\gamma} v_{n}(x, \lambda t)-v_{n}(x, t) \leq\left(\lambda^{\gamma}-1\right)\|u(x, \delta)\|_{L^{\infty}\left(R^{N}\right)} .
$$

Divided (8) by $\lambda-1$ and let $\lambda \rightarrow 1^{+}$, we get

$$
\left|\gamma v_{n}(x, t)+t v_{n t}(x, t)\right| \leq \gamma\|u(x, \delta)\|_{L^{\infty}\left(R^{N}\right)} .
$$

This inequality implies

$$
\left|u_{t}(x, t+\delta)\right| \leq \frac{C\left\||u(x, \delta) \||_{L^{\infty}\left(R^{N}\right)}\right.}{t}
$$

Let $\delta \rightarrow t$, we get the first estimate of (4).

We now prove the second estimate of (4). Notice that for fixed $t \in(0, T) u(x, t)$ is a solution of the following elliptic equations

$$
\operatorname{div}\left(|\nabla u|^{p-2} \nabla u\right)=u_{t}(x, t) \quad x \in R^{N} .
$$

By [T], there exist constants $\beta \in(0,1), C>0$ dependent only on $\left|u_{t}\right|_{L^{\infty}},|u|_{L^{\infty}}$ such that

$$
\left|\nabla u\left(x_{1}, t\right)-\nabla u\left(x_{2}, t\right)\right| \leq C\left|x_{1}-x_{2}\right|^{\beta}
$$


We now prove that $\nabla u$ is Hölder cotinuous to t. For convernience, we assume that $u$ is a smooth solution, otherwise by uniqueness of solution we can consider the regularized problem. Take the $x_{j}$-derivative in (1) to obtain

$$
\frac{\partial u_{x_{j}}}{\partial t}=\left(\operatorname{div}\left(|\nabla u|^{p-2} \nabla u\right)\right)_{x_{j}}
$$

Let $x_{0} \in R^{N}, 0<t_{1} \leq t_{2}, \Delta t=t_{2}-t_{1}, B(\Delta t)=B_{(\Delta t)^{\delta}}\left(x_{0}\right)$. Integrating (10) over $B(\Delta t) \times\left(t_{1}, t_{2}\right)$ and by integrating by parts, we get

$$
\begin{aligned}
& \int_{B(\Delta t)}\left(u_{x_{j}}\left(x, t_{2}\right)-u_{x_{j}}\left(x, t_{1}\right)\right) d x \\
= & \int_{t_{1}}^{t_{2}} \int_{\partial B(\Delta t)}\left(\operatorname{div}\left(|\nabla u|^{p-2} \nabla u\right)\right)_{x_{j}} d x d t \\
= & \int_{t_{1}}^{t_{2}} \int_{\partial B(\Delta t)} d i v\left(|\nabla u|^{p-2} \nabla u\right) \nu_{j} d \sigma d t \\
= & \int_{t_{1}}^{t_{2}} \int_{\partial B(\Delta t)} u_{t} \nu_{j} d \sigma d t .
\end{aligned}
$$

where $\nu=\left(\nu_{1}, \nu_{2}, \ldots, \nu_{N}\right)$ is the unit outward normal vector of $\partial B(\Delta t)$. By the mean value theorem, there exists $x^{*} \in B(\Delta t)$ such that

$$
\left|u_{x_{j}}\left(x^{*}, t_{2}\right)-u_{x_{j}}\left(x^{*}, t_{1}\right)\right| \leq C(\Delta t)^{1-\delta} .
$$

Combining (9) and (12) and taking $\delta=\frac{1}{1+\beta}$, we get

$$
\begin{aligned}
& \left|u_{x_{j}}\left(x_{0}, t_{2}\right)-u_{x_{j}}\left(x_{0}, t_{1}\right)\right| \leq\left|u_{x_{j}}\left(x_{0}, t_{2}\right)-u_{x_{j}}\left(x^{*}, t_{2}\right)\right| \\
& +\left|u_{x_{j}}\left(x^{*}, t_{2}\right)-u_{x_{j}}\left(x^{*}, t_{1}\right)\right|+\left|u_{x_{j}}\left(x^{*}, t_{1}\right)-u_{x_{j}}\left(x_{0}, t_{1}\right)\right| \leq C(\Delta t)^{\frac{\beta}{1+\beta}} .
\end{aligned}
$$

Therefore $u_{x_{j}} \in C_{l o c}^{\beta, \frac{\beta}{1+\beta}}\left(R^{N}\right)$ and Theorem 1 is proved.

REMARK 1. If the initial value $u_{0}$ is bounded, Theorem 1 holds for $u_{0}$ of variable sign. In fact if $\mathrm{u}$ is a solution of (1), by the uniqueness of solution $v=u+\left\|u_{0}\right\|_{L^{\infty}\left(R^{N}\right)}$ is a nonnegative solution of (1) with initial value $u_{0}+\left\|u_{0}\right\|_{L^{\infty}\left(R^{N}\right)}$. Thus Theorem 1 holds for $v$, so does $u$.

REMARK 2. For the first boundary value problem, similar theorem holds.

Consider the following problem

$$
\begin{cases}\frac{\partial u}{\partial t}=\operatorname{div}\left(|\nabla u|^{2} \nabla u\right) & (x, t) \in \Omega \times(0, T) \\ u(x, t)=\psi(x, t) & (x, t) \in \partial \Omega \times(0, T) \\ u(x, 0)=u_{0}(x) & x \in R^{N}\end{cases}
$$

where $\Omega \in R^{N}$ is a smooth bounded region.

TheOREM 2. Let $u_{0} \in L^{\infty}\left(R^{N}\right), \quad \psi, \psi_{t} \in L^{\infty}(\partial \Omega \times(0, T))$. Then the solution $u$ of (13) satisfies

$$
\left|u_{t}(x, t)\right| \leq \frac{C}{t}, \quad \nabla u \in C_{l o c}^{\beta, \frac{\beta}{1+\beta}}(\Omega \times(0, T))
$$


Proof. Without loss of generality, we assume $u \geq 0$, and $u$ large enough, otherwise replace $u$ by $u+C, C>\|u\|_{L^{\infty}}$. Set

$$
v(x, t)=\lambda^{\gamma} u(x, \lambda t), \quad \lambda>1, \quad \gamma=\frac{1}{p-2} .
$$

Then $v$ is the solution of (13) with

$$
v(x, t)=\lambda^{\gamma} \psi(x, \lambda t)(x, t) \in \partial \Omega \times(0, T), \quad v(x, 0)=\lambda^{\gamma} u_{0}(x) \quad x \in R^{N} .
$$

Notice that if $\lambda-1$ is small enough, $\psi$ large enough, we have

$$
\begin{aligned}
& \lambda^{\gamma} \psi(x, \lambda t)-\psi(x, t)=\left(\lambda^{\gamma}-1\right) \psi(x, \lambda t)+\psi_{t}(x, \xi)(\lambda-1) t \\
& =(\lambda-1)\left(\frac{\lambda^{\gamma}-1}{\lambda-1} \psi(x, \lambda t)+t \psi_{t}(x, \xi)\right)>0 .
\end{aligned}
$$

By comparison principle $\lambda^{\gamma} u(x, \lambda t) \geq u(x, t)$. Hence similar to the proof in Theorem 1 , we can prove Theorem 2 .

\section{REFERENCES}

[C] CHEN YAZHE, Hölder continuity of the gradient of the solutions of certain degenerate parabolic equations, Chin. Ann. Math., 8B(3) (1987), pp. 343-356.

[DF] DiBENEDETTO E. AND FRIEDMAN A., Hölder estimates for nonlinear degenerate parabolic systems, J. Reine Angew. Math., 357(1985), pp. 1-22.

[DH] DiBenedetTo E. AND HeRreRo M.A., On the Cauchy problem and initial traces for a degenerate parabolic equations, Trans. Amer. Soc., 314(1)(1989), pp. 187-224.

[T] PETER TOLKSDORF, Regularity for a more general class of quasilinear elliptic equations, J. Diff. Equa., 51 (1984), pp. 126-150.

[WZYL] Wu Zhuoqun, Zhao Junning, Yin Jingxue and Li Huilai, Nonlinear diffusion equations, Publishing House of Jilin University, 1996. 